\title{
MONITORIZACIÓN DE REBAÑOS DE BOVINOS A TRAVÉS DE REDES DE SENSORES INALÁMBRICOS
}

\author{
WIRELESS SENSOR NETWORKS FOR BOVINE HERD MONITORING
}

\author{
Arce, A.I.C. ${ }^{1 A}$, A.R.B. Tech ${ }^{1 \mathrm{~B}}$, A.C.S. Silva ${ }^{1 \mathrm{C}}$ e E.J.X. Costa ${ }^{1 \mathrm{D}}$
}

${ }^{1}$ Faculdade de Zootecnia e Engenharia de Alimentos. Universidade de São Paulo (FZEA-USP). Cam-

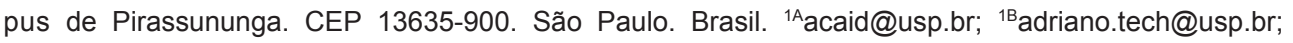
1Canac_ss@yahoo.com.br. Correspondência: 1Dernane@usp.br

\section{Palabras clave adicionales}

Telemetría.

\section{RESUMEN}

Se presenta el prototipo de un sistema proyectado utilizando técnicas de instrumentación electrónica y tecnología de comunicación inalámbrica pudiendo ser utilizado para recolectar datos fisiológicos y monitorizar rebaños de bovinos.

La necesidad de desarrollar técnicas de adquisición de datos, que causen menos perturbaciones al comportamiento natural de los animales, de interés en la zootecnia de precisión, fue la principal motivación de este trabajo. Para probar la aplicabilidad de la tecnología de las redes de sensores inalámbricos en este campo, se construyó una infraestructura utilizando técnicas de instrumentación electrónica y comunicación por radio-frecuencia. La red fue proyectada con el protocolo floating base sensor network (FBSN) que implementa una topología adhoc con selección aleatoria de nodos. Los prototipos construidos fueron utilizados para efectuar colectas de datos de la actividad eléctrica cerebral y temperatura corporal de seis novillas de raza Holandesa.

A fin de optimizar la infraestructura, fue desarrollado un software que a partir de la simulación del desplazamiento de un rebaño bovino ayuda a determinar la mejor distribución de esa infraestructura en el área monitorizada.

Los resultados alcanzados mostraron que es posible utilizar redes de sensores inalámbricas como técnicas eficientes para obtener datos fisiológicos de bovinos.

\section{SUMMARY}

This paper presents a prototype system for

\section{AdDitional KEYWORDS}

Telemetry.

monitoring bovine herds developed using electronic instrumentation and wireless techniques. It is able to acquire physiological data for ethological studies.

The main stimulus of this work was the precision animal production researcher's interest in developing and testing new techniques that could allow data acquisition with less interference in animal behavior. In order to test the feasibility of the wireless sensor network technology in this area, an infrastructure was developed using electronic instrumentation and radio frequency wireless communication techniques. The network was projected to support the floating base sensor network protocol that is a special kind of adhoc topology with randomic selection of path nodes. The developed prototypes were used to acquire brain electrical activity and body temperature from bovines.

In order to optimize the spatial distribution of the wireless networks infrastructure, a software to simulate herd displacement was developed for assist the achievement of minimum-cost for antennas distribution.

The results obtained prove that the wireless sensor networks can be considered as an efficient technique to be used to acquire bovine's physiological data.

\section{INTRODUCCIÓN}

Las normalizaciones internacionales con respecto a los productos de origen animal, enfocan cada vez más la calidad al control de los animales antes de su sacrificio (Aguiar 
y Silva, 2002). Dentro de ese proceso de control antes del sacrificio de los animales, se encuentra la monitorización de varios datos fisiológicos así como el acompañamiento minucioso del bovino, desde su nacimiento hasta su sacrificio (Barcellos y Ferreira, 2003).

Entre los varios factores que interfieren en la productividad animal, tales como la genética y la nutrición, los factores bioclimatológicos y ambientales requieren especial atención de los científicos, debido a que las características climatológicas de cada región pueden causar estrés e incomodo térmico a los animales durante períodos de elevadas temperaturas, resultando en pérdidas productivas debido a la reducción de la ingestión de alimentos, reducción del incremento de peso y, en casos extremos, la muerte de los animales (Mader et al., 2002; Brown-Brandl et al., 2003; Hahn, 1999).

En estas circunstancias, los criadores de ganado y los científicos que estudian el comportamiento de esos animales, necesitan adquirir datos a partir de experimentos etológicos que les permitan entender como los animales responden a los cambios ambientales a fin de tomar decisiones estratégicas.

La adquisición de datos de temperaturas timpánica, rectal y corporal (Mader et al., 2002; Hahn, 1999) así como la medición de las frecuencias cardiaca y respiratoria (Brown-Brandl et al., 2003) y la actividad eléctrica cerebral (Suzuki et al., 1990), son prácticas utilizadas en los estudios etológicos y deben ser hechas de preferencia, sin interferir en el comportamiento natural de los animales estudiados. Sin embargo, la adquisición de estos datos presenta ciertas dificultades técnicas con relación al ambiente en que se desarrolla el experimento. Normalmente, el animal estudiado tiene que ser retirado de su área de cría y confinado en un local donde se realiza la obtención de los datos. Según Brockway e Hassler (1993), el uso de métodos de confinamiento durante la experimentación, a pesar de permitir que se adquieran datos de los animales conscientes, puede introducir artefactos relativos al estrés ocasionado por la manipulación. Estos autores afirman también que minimizar o eliminar las fuentes causantes de estrés externo es crucial para entender el proceso que está siendo evaluado. Por este motivo, es necesario el uso de un sistema telemétrico ya que la radio-telemetría permite la medida de variables biológicas de los animales conscientes y que pueden moverse libremente (Brockway y Hassler, 1993). El mayor aporte del empleo de esta metodología es que el animal no sufre de estrés que podría ser causado por el confinamiento o por la presencia humana durante la obtención de datos (Gacsalyi et al., 2000).

La monitorización animal por telemetría demanda el desarrollo de equipos para la adquisición de datos específicos para este tipo de trabajo. El avance de las investigaciones en el área de redes de sensores inalámbricos, que son básicamente sistemas telemétricos, posibilitó la aplicación de esta tecnología en la toma de datos de animales y su empleo es viable según Kettlewell et al. (1997) y Silva et al. (2005).

Las redes de sensores inalámbricos son actualmente utilizadas para monitorizar y controlar varios tipos de ambientes, inclusive de sistemas biológicos, y aumentan la precisión de los datos adquiridos (Asada et al., 2000).

Existen varios obstáculos en el desarrollo de sistemas con redes inalámbricas para este tipo de actividad, por ejemplo: la limitación de energía, la capacidad computacional, reducción del tamaño de los sensores y la minimización del costo de la infraestructura final.

Este trabajo muestra una aplicación de técnicas de instrumentación electrónica y comunicación inalámbrica en el desarrollo de una infraestructura de red de sensores, específica para monitorizar variables fisiológicas de bovinos. El empleo de la metodología aquí expuesta podrá contribuir al perfeccionamiento de técnicas de obtención de 
datos de animales que introduzcan menos errores sistemáticos durante la experimentación.

\section{METODOLOGÍA}

\section{ESTRUCTURA DE LAS REDES INALÁMBRICAS}

Una red de sensores inalámbricos (WSN - Wireless Sensor Network) es una red de pequeños nodos computacionales con sensores y dispositivos de transmisión telemétricos debidamente acoplados (Min etal., 2002).

El protocolo Floating Base Sensor Network (FBSN) propuesto por Silva et al. (2005), es un protocolo de comunicación de redes inalámbricas, cuya principal característica es poseer nodos móviles con capacidad de convertirse en una estación radio base temporaria (ERBT). En ese protocolo, el algoritmo de control rige dinámicamente cómo los nodos irán a comunicarse en la red, eligiendo de forma aleatoria estaciones radio base móviles, que puedan ser conectadas a través de una de las estaciones radio base fijas (ERBF). La figura 1 muestra el esquema de una red FBSN cuyo algoritmo puede ser visto en la figura 2.

La ERBF puede establecer comunica- ción directa solamente con aquellos nodos móviles que estén dentro del área de cobertura. El área de cobertura es el área del círculo, con centro en la ERBF, dentro del cual es posible establecer una comunicación bilateral entre la ERBF y el nodo móvil; su dimensión depende, por tanto, del radio de cobertura de ambos dispositivos.

\section{ARQUITECTURA BÁSICADEL HARDWARE}

El sistema descrito aquí, consta de dos módulos principales; el primero es responsable del acondicionamiento y transmisión de la señal recolectada (nodos sensores) y el segundo recibe los datos y los transfiere a un sistema informatizado (estaciones radio base fijas ERBF).

\section{Nodos sensores}

Estos módulos, llevados por los animales, están constituidos por cuatro partes:

1 - Acondicionamiento de señales.

2-Conversión analógico-digital.

3- Transmisión de datos digitales.

4- Bus I2C.

Los nodos sensores fueron equipados con un amplificador de señales cerebrales que fue proyectado específicamente para este tipo de aplicación.

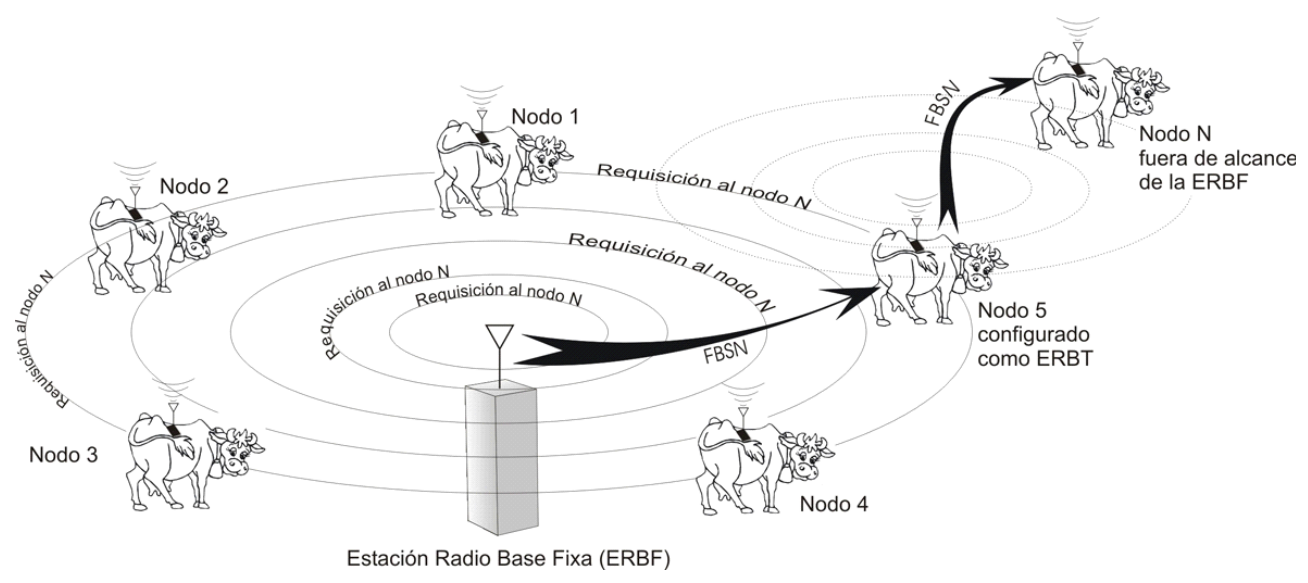

Figura 1. Esquema de una red FBSN (Silva et al., 2005). (FBSN Network Scheme (Silva et al.,2005)). 


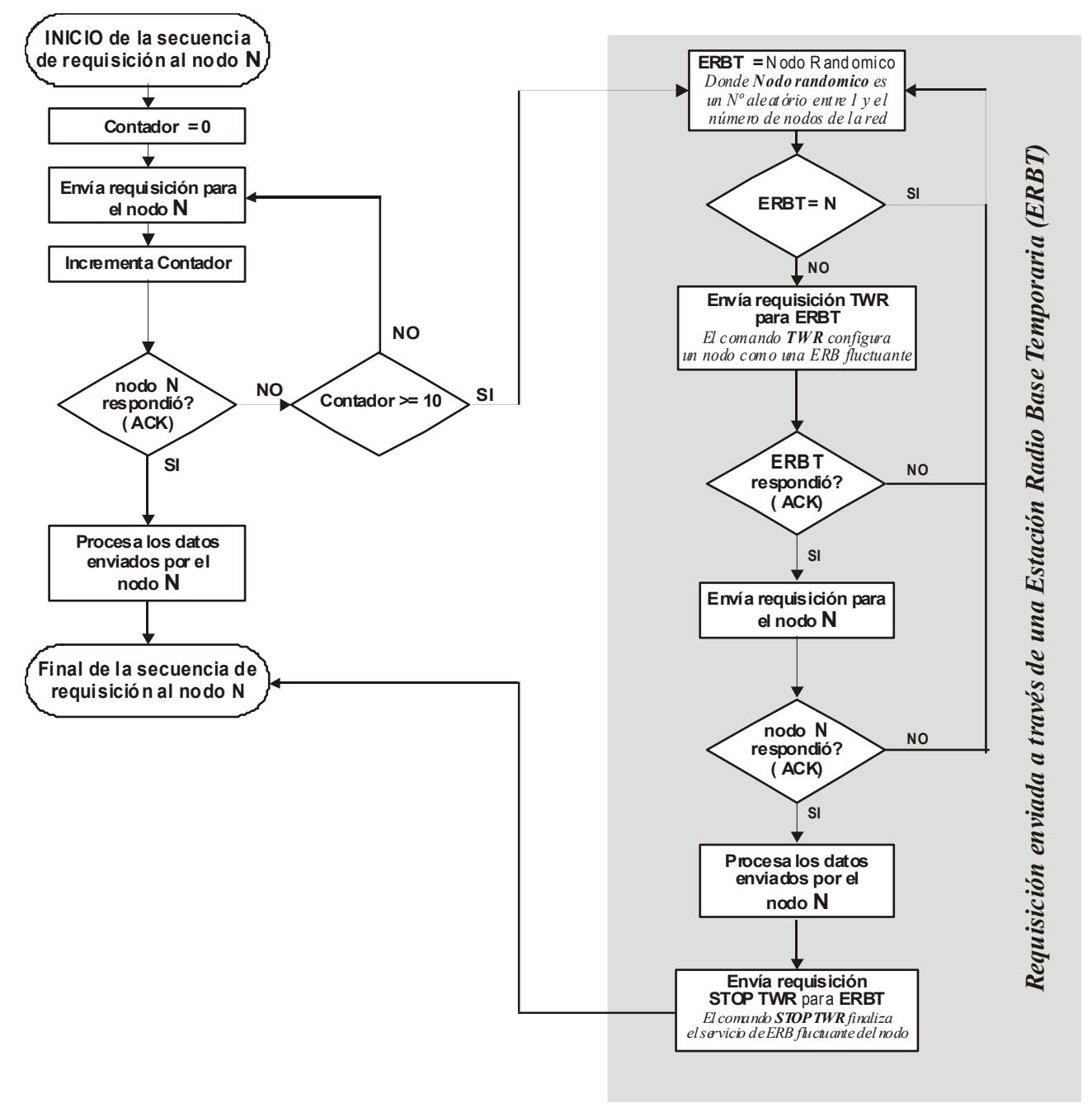

Figura 2. Algoritmo de control de la FBSN. (FBSN control algorithm).

\section{Acondicionamiento de señales}

Las señales obtenidas a partir de transductores eléctricos son amplificadas utilizando amplificadores operacionales y filtros activos y pasivos.

Fueron utilizados electrodos para recolección de señales cerebrales.

\section{Conversión analógico-digital}

Las señales analógicas acondicionadas son convertidas en datos digitales utilizan- do el conversor A/D de 12 bits de un microcontrolador PIC-16C774 Microchip ${ }^{\circledR}$ que también es responsable de conmutar entre dos o más señales en caso de existir más de un sensor acoplado al módulo y controlar el protocolo FBSN.

\section{Transmisión de datos digitales}

E1 transceptor BIM2-433-160 Radiometrix ${ }^{\circledR}$ fue escogido para esta tarea por su bajo consumo y tamaño reducido. Este dis- 
positivo tiene la capacidad de transmitir y recibir datos a través de radio-frecuencia y fue acoplado directamente al microcontrolador PIC-16C774 y opera en FM a 433 Mhz.

\section{Bus 12C}

I2C es un protocolo sincrónico idealizado por Philips ${ }^{\circledR}$ que soporta direccionamiento de dispositivos y utiliza un bus bidireccional simple de dos líneas (Línea de clock o SCL y línea de datos o SDK). Varios circuitos conectados al mismo bus pueden comunicarse usando este protocolo.

El microcontrolador PIC-16C774 fue programado para controlar el flujo de datos en el bus I2C y recibir informaciones de los sensores digitales acoplados.

Fueron utilizados en el prototipo, sensores de temperatura digitales TMP100 Texas Instruments ${ }^{\circledR}$.

\section{Estaciones radio base fijas (ERBF)}

Este módulo es responsable de la recepción de los datos enviados por los nodos sensores y retransmite esa información hasta alcanzar a la estación central, a través de una infraestructura de ERBF's distribuidas en el área monitorizada como se muestra en la figura 3.

La figura 4 muestra el diagrama esquemático de una ERBF. En la construcción del prototipo, fue usado el transceptor BIM2433-160 acoplado a un microcontrolador PIC-16F877.

Una de las ERBF's fue conectada a un microcomputador cuyo papel es el de coor-

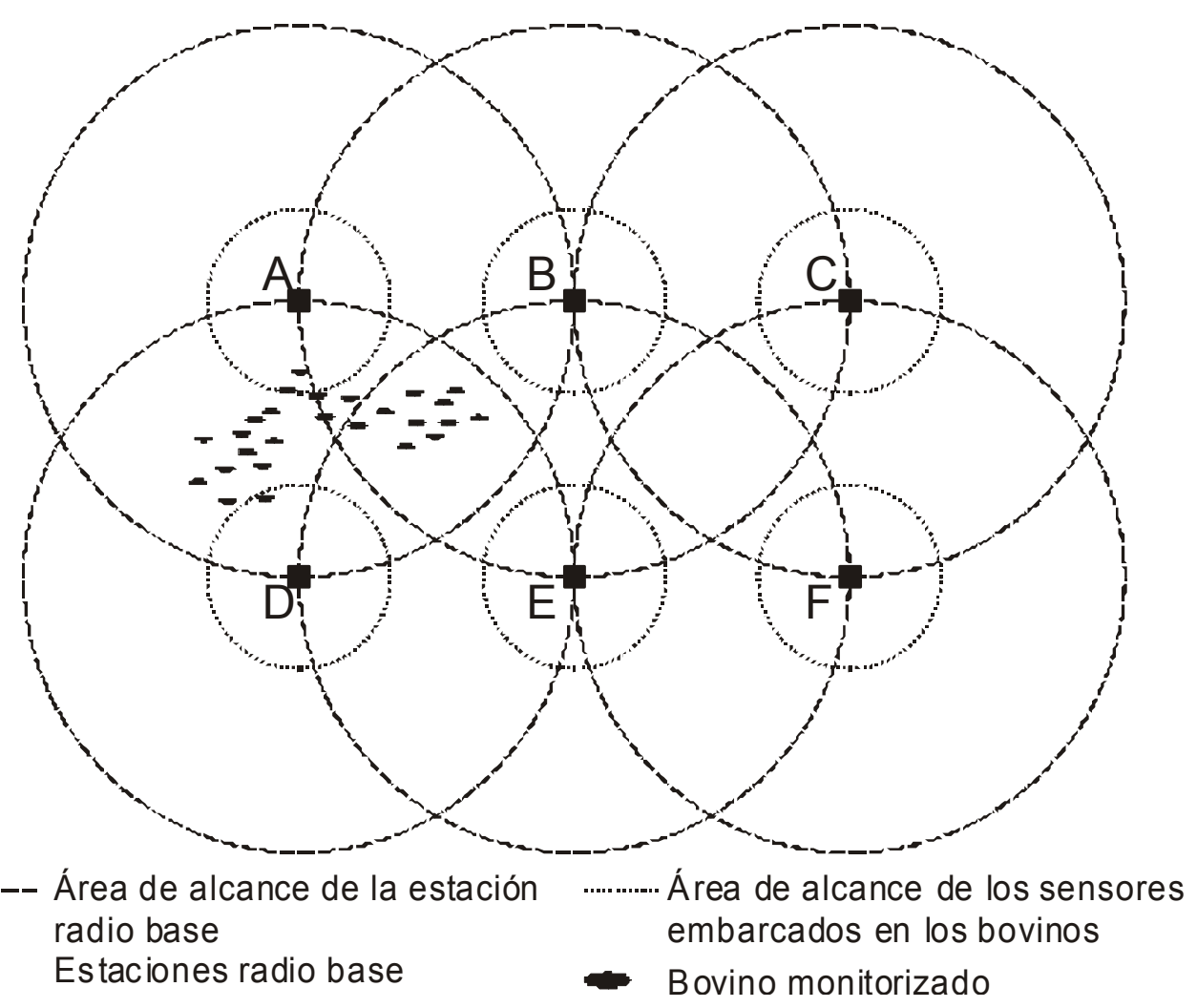

Figura 3. Infraestructura de monitorización. (Illustration of monitoring infrastructure). 


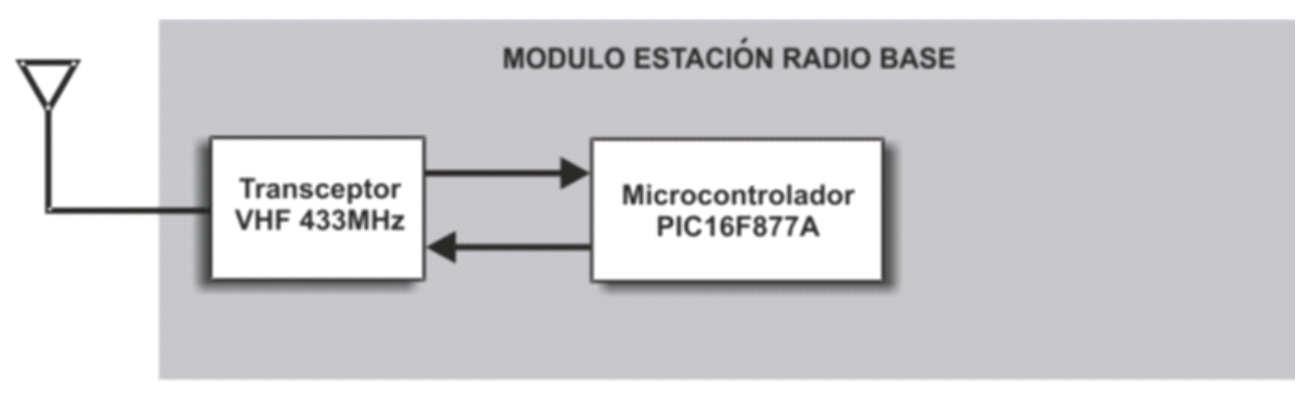

Figura 4. Diagrama esquemático de las ERBF. (ERBF Schematics).

dinar el flujo de datos de la red a través del protocolo FBSN.

\section{EXPERIMENTACIÓNCONANIMALES}

Fueron realizados dos experimentos con duración de una semana, en la Facultad de Zootecnia e Ingeniería de Alimentos de la Universidad de São Paulo $\left(21^{\circ} 59^{\prime} \mathrm{S}, 47^{\circ} 27^{\prime} \mathrm{W}\right)$. Se utilizaron seis novillas de raza Holande$\mathrm{sa}$, con $528 \pm 28 \mathrm{~kg}$, y con aproximadamente 2,5 años, en condiciones normales de salud. Ambos experimentos se realizaron de acuerdo con la bioética de la Universidad de São Paulo y el procedimiento fue acompañado por el médico veterinario de la Facultad.

Para el $1^{\mathrm{er}}$ experimento, los nodos sensores se instalaron usando bolsas impermeables adheridas al dorso de los animales.
Los electrodos para adquisición de señales cerebrales fueron implantados según los procedimientos descritos por Silva et al. (2005). La figura 5a muestra una de las novillas llevando el equipo durante el primer experimento.

Para el $2^{\circ}$ experimento, fue fabricado un cabestro con una bolsa impermeable donde se acondicionaron los nodos sensores.

Los nodos transceptores fueron programados para realizar captura de EEG a una frecuencia de $120 \mathrm{~Hz}$ y la red colectó datos de los animales a través del envío de comandos de requisición a los nodos sensores. La figura 5b muestra la imagen de un animal equipado con un nodo sensor durante el segundo experimento.

En ambos experimentos, inmediatamen-
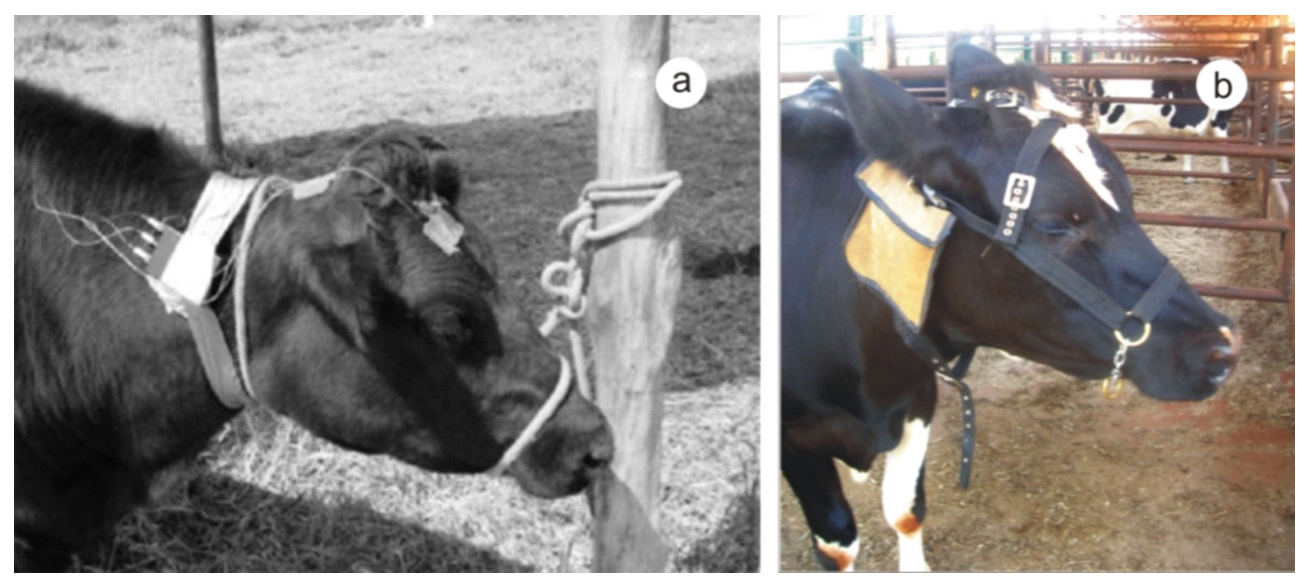

Figura 5. Animal equipado con un nodo sensor. (Animal carrying a sensor node).

Archivos de zootecnia vol. 58, núm. 222, p. 258. 
te después de la implantación de los equipos, los animales fueron soltados en un área experimental provista de la infraestructura de monitorización.

Para validar los datos de EEG adquiridos con los equipamientos fabricados, fue verificado si las frecuencias presentes en trechos libres de artefactos de los datos obtenidos pertenecen a los intervalos de 2 a 7 $\mathrm{Hz}$ y de 15 a $30 \mathrm{~Hz}$ conforme los datos obtenidos por Suzuki et al. (1990).

\section{SOFTWARE SIMULADOR DE DES- PLAZAMIENTODEL REBAÑOBOVINO PARA LA OPTIMIZACIÓN DE UNA INFRAESTRUCTURAWSN}

La necesidad de optimización y reducción de costos de implementación de una infraestructura inalámbrica, fue motivo para el empleo de una herramienta que facilite la etapa de planeamiento de la infraestructura de nodos fijos.

Se trata de un simulador que al mismo tiempo representa el desplazamiento del rebaño de bovinos y simula una FBSN en actividad. De esta forma, es posible definir la infraestructura óptima para monitorear un rebaño de bovinos en función de su desplazamiento que normalmente es influenciado por obstáculos naturales o artificiales presentes en el área monitoreada tales como restos de vegetación y construcciones que pueden ser representados en la tela del simulador. Durante la simulación la dirección adoptada por el bovino líder del rebaño virtual resulta del estímulo generado por dos modelos de desplazamiento implementados y la organización del rebaño durante el desplazamiento simula los patrones naturales de desplazamiento de rebaños bovinos.

\section{PATRONESdEdesplaZAMIENTOdEL REBAÑo BOVINO}

El rebaño bovino se comporta como un grupo individualizado y jerarquizado. Dentro del rebaño se destacan dos tipos de líderes: el líder social directamente ligado a la estructura jerárquica del grupo y el líder espacial, que es el animal que tiene la iniciativa de efectuar algún movimiento en respuesta a un estímulo o en función de poseer mayor conocimiento sobre el área de cría y sus recursos (Sato, 1982; Tyler, 1972; Greenwood y Rittenhouse, 1997). El rebaño sigue naturalmente a éste último.

Durante el desplazamiento del rebaño actúan dos fuerzas opuestas que afectan individualmente a sus miembros (Paranhos, 2000). La fuerza cohesiva que tiende a juntarlos está relacionada con los diferentes niveles de asociación (parejas, gemelos, grupos filiales, etc.) determinando la distancia social $(d s)^{1}$. La fuerza repulsiva tiende a separarlos evitando que se aproximen demasiado y determina la distancia individual $(d i)^{2}$.

Con estas informaciones podemos modelar el comportamiento espacial de cada individuo dentro del rebaño como:

$$
d_{n-z}=\sqrt{\left(\left(x_{n}-x_{z}\right)^{2}+\left(y_{n}-y_{z}\right)^{2}\right)} \quad \text { [1] }
$$

$d i_{z} \leq d_{n-z} \geq d i_{n} d_{n-z} \leq d s_{n} \Leftrightarrow z$ es líder

Donde:

$d_{n-z}=$ Distancia euclidiana del animal $n$ al animal $z$; $d l_{n}^{n-z}, d i_{z}=$ Distancias individuales; $d s_{n}=$ Distancia social del animal $n$;

$x_{n}, y_{n}=$ Coordenadas cartesianas del animal $n$; $x_{z}, y_{z}=$ Coordenadas cartesianas del animal $z$.

\section{DESARROLLODEL SIMULADOR}

El problema fue modelado utilizando análisis orientado a objetos el cual ofrece una estructura conceptual muy apropiada a la modelación de sistemas biológicos (Conquillard y Hill, 1997) y propone para varios dominios de conocimiento un punto de vista unificado del sistema, permitiendo

${ }^{1}$ Podemos definir $(d s)$ como la mayor distancia a la que un individuo en condiciones normales se separa del grupo.

${ }^{2}(d i)$ Es la distancia mínima entre los animales del grupo. 


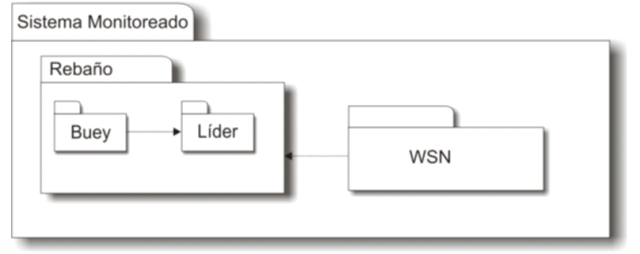

Figura 6. Diagrama UML del dominio. (Domain's UML diagram).

resolver problemas de comunicación.

La figura 6 muestra el modelo de dominio idealizado para representar el problema.

El paquete rebaño describe el conjunto de animales monitoreados por el sistema. Este paquete es compuesto a su vez por dos sub-paquetes:

1. El paquete Buey describe a los miembros del rebaño cuyas distancias espaciales son regidas por modelos basados en los parámetros de distancia individual y social. Cada uno de esos miembros representa al mismo tiempo un nodo móvil WSN.

2. El paquete líder describe a los animales del rebaño que tienen iniciativa propia de efectuar un desplazamiento.

El paquete WSN representa la infraestructura de nodos fijos o estaciones radio base necesaria para efectuar el monitoreo del rebaño.

El diseño del simulador de desplazamiento bovino tiene su enfoque en las interacciones espaciales de los animales entre sí y de los líderes ejecutando un desplazamiento.

El líder es la entidad decisiva del sistema $\mathrm{y}$ determina el desplazamiento del resto del rebaño basado en los modelos random walk ${ }^{3}$ y pseudobrowniano $0^{4}$, en cuanto su interacción espacial con el resto del rebaño es mantenida.

\section{ARQUITECTURADELSOFTWARE}

Como se observa en la figura 7, el simulador fue estructurado en 6 paquetes:

1. Paquete rebaño: derivado del análisis de dominio, incluye un paquete de eventos generados durante la simulación:

- OnBoiMove

- GeneraAnimales

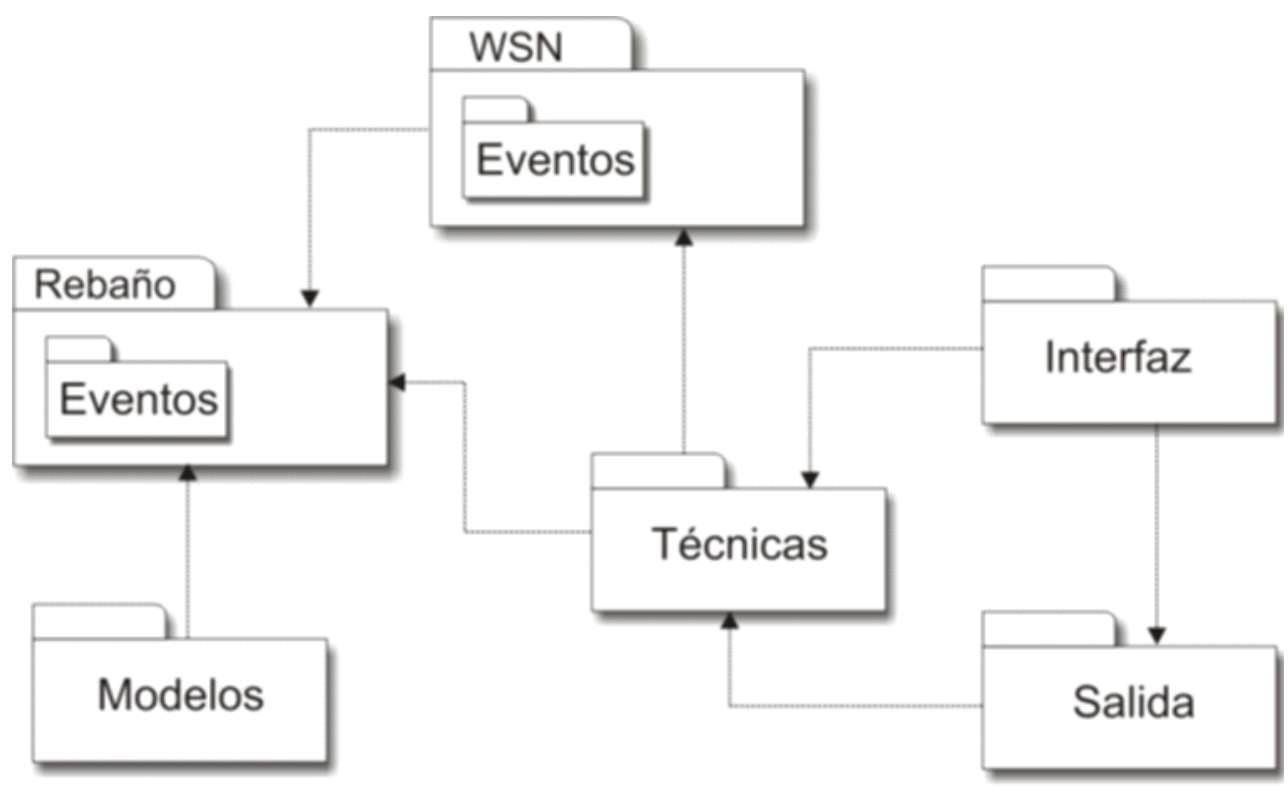

Figura 7. Arquitectura UML del simulador. (UML simulator's architecture).

Archivos de zootecnia vol. 58, núm. 222, p. 260. 
- Reorganiza

- Onrequested

2. Paquete modelos: contiene los modelos matemáticos de movimiento (random walk $^{3}$ y pseudobrowniano ${ }^{4}$ ) y los modelos de interacción espacial de los animales.

3. Paquete interfaz: conforma la interfaz gráfica para la configuración de los parámetros del sistema: número de animales del rebaño, distancia social máxima, distancia individual mínima, tamaño del paso (random walk) diseño de obstáculos en el área de desplazamiento y radio de cobertura de un nodo fijo.

4. Paquete de técnicas: contiene las clases gerenciales del kernel del simulador.

5. Paquete de salida: contiene los recursos para la salida de información del simulador así como la representación gráfica de la evolución de los procesos de simulación.

6. Paquete WSN es derivado del análisis de dominio y contiene el algoritmo de control de la FBSN.

Los parámetros iniciales requeridos por el simulador son:

- Número de animales del rebaño.

- Distancia social máxima.

- Distancia individual mínima.

- Tamaño del paso (solamente para random walk).

- Radio de cobertura de los nodos fijos.

- Tipo de movimiento (random walk o pseudobrowniano).

- Obstáculos en el área de desplazamiento ${ }^{5}$.

${ }^{3}$ Describe el desplazamiento del bovino líder utilizando un proceso estocástico simple de selección de nodos aleatorios dentro de una red simétrica. ${ }^{4}$ Modelo browniano propuesto por Albert Einstein (1905) donde en lugar del choque de moléculas sobre un corpúsculo macroscópico, el estímulo es del ambiente externo a un animal en forma de otros animales o características del área de cría tales como detalles del relieve, obstáculos, áreas ya pastadas, etc.

${ }^{5}$ Es posible diseñar en la ventana de simulación obstáculos que representen obstáculos reales que interfieren en el desplazamiento del rebaño.

\section{SIMULADORDELAREDDESENSORESFBSN}

Partiendo de los gráficos de propagación de las antenas de las estaciones radio base fijas (radio de cobertura) y de los nodos sensores, el simulador genera en el espacio de cría virtual, una distribución de nodos fijos para un rebaño unitario, o sea, un único animal en cualquier posición del área de cría estaría siempre dentro del área de cobertura de la infraestructura de nodos fijos.

Con el rebaño virtual en movimiento, es posible reducir progresivamente la cantidad de estaciones fijas (ERBF) y visualizar su efecto en el desempeño general de la red. La situación óptima es aquella en que todos los animales pueden ser alcanzados a través de la red pero pocos individuos deben estar dentro del área de cobertura de las ERBF.

\section{RESULTADOS}

Elárea mostrada en la figura 8 fue representada en la tela simulador.

Fueron configurados los parámetros para simulación del rebaño bovino como muestra la tabla I.

Una simulación de los obstáculos artificiales (cercas) y naturales (restos de floresta) presentes en el área real fue representada en la interfaz del programa. El software calculó la distribución de máximo rendimiento de las ERBF's para el área real y creó

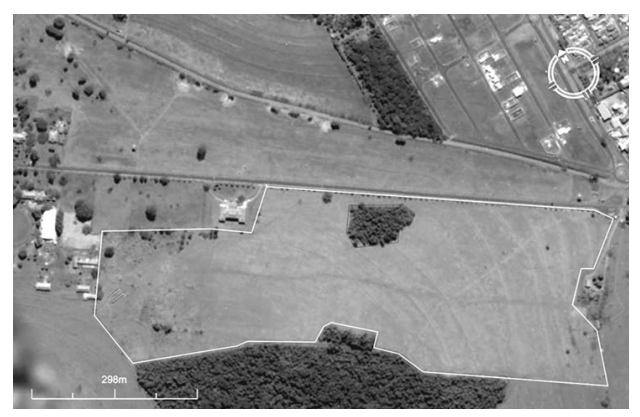

Figura 8. Área escogida para probar el simulador. (An example of area for simulator test). 
Tabla I. Parámetros utilizados en el simulador. (Input parameters for setting the simulator).

\begin{tabular}{lc}
\hline Parámetro & Valor \\
\hline Número de animales del rebaño & 100 \\
Distancia social máxima & $10 \mathrm{~m}$ \\
Distancia individual mínima & $7 \mathrm{~m}$ \\
Modelo de desplazamiento & Browniano \\
Radio de cobertura ERBF & $150 \mathrm{~m}$ \\
Cobertura de los nodos sensores & $50 \mathrm{~m}$ \\
\hline
\end{tabular}

un rebaño virtual a partir de los parámetros configurados.

Finalmente, el programa simuló el desplazamiento del rebaño virtual dentro del área real (figura 9) y así fue posible aumentar la distancia entre las ERBF's hasta alcanzar la configuración óptima.

Con base en los resultados obtenidos de la simulación, fueron distribuidas antenas en el área real donde fueron soltadas las novillas equipadas con los nodos sensores. Con la infraestructura implementada, fue posible realizar el registro de la actividad eléctrica cerebral (EEG) de los bovinos totalmente sueltos en el área de pastoreo.

Como los animales estaban totalmente libres, la señal de EEG presentó gran cantidad de artefactos oriundos de movimientos bruscos de los animales. A pesar de eso, fue posible obtener varios trechos libres de

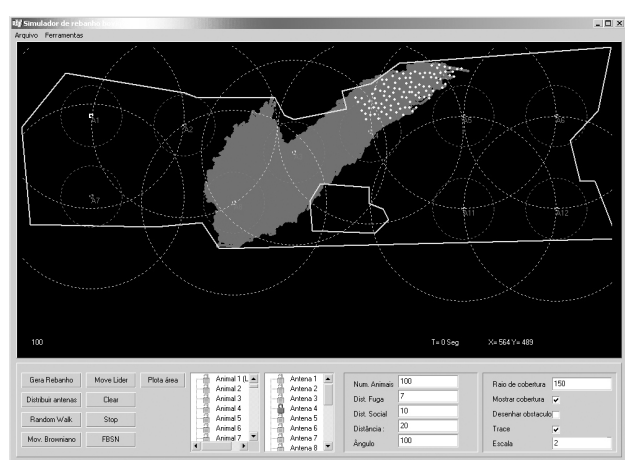

Figura 9. Rebaño virtual en movimiento. (Screenshot of the virtual herd movement). artefactos con $5 \mathrm{~s}$ de duración a partir de los datos recolectados. La literatura, relativa a EEG de bovinos reporta el uso de trechos de datos con duración entre $5 \mathrm{~s}$ (Merrick y Scharp, 1971) y $8,5 \mathrm{~s}$ (Jones and Pettitt, 1992), por este motivo, los trechos obtenidos fueron considerados adecuados para procesamiento. Las frecuencias encontradas $(2-10 \mathrm{~Hz} ; 13$ $27 \mathrm{~Hz}$ ) están de acuerdo con las obtenidas por Suzuki et al. (1990) en becerros.

Fue verificado, durante los experimentos con animales, que estos toleraron la presencia de los equipamientos, principalmente cuando se utilizó el cabestro para acondicionamiento de los nodos sensores, y se pudo observar que no modificaron sus hábitos normales de alimentación y rumia durante los experimentos.

\section{CONCLUSIONES}

La red de sensores inalámbricos proyectada, posibilitó la implementación de una infraestructura que permitió monitorear rebaños de bovinos y adquirir de los animales datos biológicos.

Considerando que los trechos de EEG obtenidos a través de la red de sensores inalámbricos al ser analizados presentaron resultados coincidentes con los datos encontrados en la literatura, podemos concluir que este sistema fue eficiente para la obtención de datos de EEG y puede ser usado para adquirir otros datos biológicos de bovinos en experimentos etológicos.

El comportamiento natural de los animales durante los experimentos respecto a sus hábitos de pastaje y ruminación nos permitió inferir la ausencia de estrés en estos durante la toma de datos. Esta reducción de los factores causadores de estrés se dio principalmente gracias a que la metodología empleada permitió la monitorización de los animales libres en un área experimental sin interferencia humana directa.

El empleo del simulador fue de singular importancia ya que permitió definir una buena distribución de la infraestructura de

Archivos de zootecnia vol. 58, núm. 222, p. 262. 


\section{MONITORIZACIÓN DE REBAÑOS CON REDES DE SENSORES INALÁMBRICOS}

monitorización y podrá en el futuro viabilizar técnicas de monitorización de rebaños numerosos en grandes áreas de cría extensiva.

El resultado de los experimentos, por lo tanto, mostró que las redes de sensores inalámbricas son soluciones eficientes, aplicables a la zootecnia de precisión y pueden ser utilizadas para la monitorización de variables fisiológicas de bovinos.

Esta técnica está siendo utilizada actualmente para adquirir datos de la actividad

\section{BIBLIOGRAFÍA}

Aguiar, D.R.D. and A.L. Silva. 2002. Changes in beef consumption and retailing competitiveness in Brazil: a rapid appraisal. Agribusiness, 18: 145-161.

Asada, G., I. Bhatti, T.H. Lin, S. Natkunanthanan, F. Newberg, R. Rofougaran, A. Sipos, S. Valoff, G.J. Pottie and W.J. Kaiser. 2000. Wireless Integrated Network Sensors (WINS). Proc. SPIE, 3673: 11-18.

Barcellos, M.D. e A.G.C. Ferreira. 2003. Análise de marcas em carne bovina. Agroanalysis, 23: $27-$ 30 .

Brown-Brandl, T.M., J.A. Nienaber, R.A. Eingenberg, G.L. Hahn and H. Freetly. 2003. Termoregulatory responses of feeder cattle. J. Therm. Biol., 28: 149-157.

Brockway, B.P. and C.R. Hassler. 1993. Aplication of radiotelemetry to cardiovascular measurements in pharmacology and toxicology. In: Salem, H.; Basken, S.I. (Eds). New technologies and concepts for reducing drug toxities. CRC Press. Boca Raton. p.109-132.

Conquillard, P. et D.R.C. Hill. 1997. Modelisation et simulation des ecosystemes. Masson. Paris.

Greenwood, P.T. and L.R. Rittenhouse. 1997. Feeding area selection: the leader-follower phenomena. Proc. West. Sect. Am. Soc. Anim. Sci., 48: 267-269.

Gacsalyi, U., R. Zabielski and S.G. Pierzynowski. 2000. Telemetry facilitates long-term recording of gastrointestinal myoelectrical activity in pigs. Exp. Physiol., 85: 239-241.

Hahn, G.L. 1999. Dynamic responses of cattle to thermal heat loads. J. Anim. Sci., 77: 10-20.

Jones, P.N. and A.N. Pettitt. 1992. Comparison of EEGs before and after stunning of cattle taking eléctrica cerebral de bovinos que permitan identificar situaciones de estrés térmico en bovinos adultos de la raza Holandesa y que posibiliten estudiar la adaptación de estos animales a las variadas condiciones ambientales de producción mucho antes de la aparición de respuestas fisiológicas típicas. Esos estudios podrán resultar en la identificación de parámetros de confort más precisos para la cría de las razas que están siendo estudiadas.

account of animal-to-animal variation. $J$. Biomedicine, 34: 815-825.

Kettlewell, P.J., M.A. Mitchell and I.R. Meeks. 1997. An implantable raiotelemetry system for remote monitoring of heart rate and deep body temperature in poultry. Comput. Electron. Agr., 17: 161-175.

Mader, T.L., S.M. Holt, G.L. Hahn, M.S. Davis and D.E. Spiers. 2002. Feeding strategies for managing heat load in feedlot cattle. J. Anim. Sci., 80: 2373-2382.

Merrick, A.W. and D.W. Scharp. 1971. Electroencephalography of resting behavior in cattle, with observations on the question of sleep. Am. J. Vet. Res., 32: 1993-1997.

Min, R.B., M. Cho, S. Ickes, N. Shih, E. Sinha, A. Wang and A. Chandrakasan. 2002. Energycentric enabling technologies for wireless sensor networks. IEEE Wireless Communications. 9: 28-37.

Paranhos da Costa, M.J.R. 2000. Ambiência na produção de bovinos de corte a pasto. Anais de Teología, 18: 26-42.

Sato, S. 1982. Leadership during actual grazing in a small herd of cattle. Appl. Anim. Ethol., 8: 53-65.

Silva, A.C.S., A.C. Arce, S. Souto and E.J.X. Costa. 2005. A wireless floating base sensor network for physiological response to livestock. Comput. Electron. Agr., 49: 246-254.

Suzuki, M., K. Sitizyo and T. Takeuchi. 1990. Electroencephalogram of Japanese black calves affected with cerebrocortical necrosis. Jpn. J. Vet. Sci., 52: 1077-1087.

Tyler, S.J. 1972. The behaviour and social organization of the New Forest ponies. Anim. Behav. J. Monographs, 5: 87-196. 